\title{
3. \\ Über Integrale der hydrodynamischen Gleichungen, welche den Wirbelbewegungen entsprechen.
}

\author{
(Von Herrn H. Helmholtz.)
}

E $_{\mathrm{s}}$ sind bisher Integrale der hydrodynamischen Gleichungen fast nur unter der Voraussetzung gesucht worden, dafs die rechtwinkligen Componenten der Geschwindigkeit jedes Wassertheilchens gleich gesetzt werden können den nach den entsprechenden Richtungen genommenen Differentialquotienten einer bestimmten Function, welche wir das Geschwindigkeitspotential nennen wollen. Allerdings hat schon Lagrange ${ }^{*}$ ) nachgewiesen, dafs diese Voraussetzung zulässig ist, so oft die Bewegung der Wassermasse unter dem Einflusse von Kräften entstanden ist und fortgesetzt wird, welche selbst als Differentialquotienten eines Kräflepotentials dargestellt werden können, und dafs auch der Einflufs bewegter fester Körper, welche mit der Flüssigkeit in Berührung kommen, die Gültigkeit jener Voraussetzung nicht abändert. Da nun die meisten mathematisch gut definirbaren Naturkräfte als die Differentialquotienten eines Kräftepotentials dargestellt werden können, so fallen auch bei weitem die meisten mathematisch zu behandelnden Fälle von Flüssigkeitsbewegung in die Zahl derer, bei denen ein Geschwindigkeitspotential existirt. Indessen hat schon Euler **) darauf aufmerksam gemacht, dafs es doch auch Fälle von Flüssigkeitsbewegung giebt, in denen kein Geschwindigkeitspotential existirt, z. B. die Drehung einer Flüssigkeit um eine Axe mit gleicher Winkelgeschwindigkeit aller Theilchen. Zu den Kräften, welche solche Arten von Bewegungen hervorbringen können, gehören magnetische Kräfte, welche auf eine von electrischen Strömen durchlaufene Flüssigkeit wirken, und namentlich die Reibung der Flüssigkeitstheilchen an einander und an festen Körpern. Der Einflufs der Reibung auf Flüssigkeiten konnte bisher noch nicht mathematisch definirt werden, und doch ist derselbe in allen Fällen, wo es sich nicht um unendlich kleine Schwingungen handelt, sehr grofs, und bringt

*) Mécanique analytique. Paris 1815. T. II, p. 304.

**) Histoire de l'Acad. des Sciences de Berlin. An. 1755, p. 292.

Journal für Mathematik Bd. LV. Heft 1. 
die bedeutendsten Abweichungen zwischen der Theorie und der Wirklichkeit hervor. Die Schwierigkeit diesen Einflufs zu definiren, und Methoden zu seiner Messung zu finden, beruhte zum grofsen Theile wohl auch darin, dafs man keine Anschauung von den Formen der Bewegung hatte, welche die Reibung in der Flüssigkeit hervorbringt. In dieser Beziehung schien mir daher eine Untersuchung der Bewegungsformen, bei denen kein Geschwindigkeitspotential existirt, von Wichtigkeit zu sein.

Die folgende Untersuchung wird nun lehren, dafs in den Fällen, wo ein Geschwindigkeitspotential existirt, die kleinsten Wassertheilchen keine Rotationsbewegungen haben, wohl aber ist wenigstens ein Theil der Wassertheilchen in Rotation begriffen in solchen Fällen, wo kein Geschwindigkeitspotential existirt.

Wirbellinien nenne ich Linien, welche durch die Flüssigkeitsmasse so gezogen sind, dafs ihre Richtung überall mit der Richtung der augenblicklichen Rotationsaxe der in ihnen liegenden Wassertheilchen zusammentrifft.

Wirbelfüden nenne ich Theile der Wassermasse, welche man dadurch aus ihr herausschneidet, dafs man durch alle Puncte des Umfangs eines unendlich kleinen Flăchenelements die entsprechenden Wirbellinien construirt.

Die Untersuchung ergiebt nun, dafs wenn für alle Kräfte, welche auf die Flüssigkeit wirken, ein Kräftepotential existirt

1) kein Wassertheilchen in Rotation kommt, welches nicht von Anfang an in Rotation begriffen ist.

2) Die Wassertheilchen, welche zu irgend einer Zeit derselben Wirbellinie angehören, auch indem sie sich fortbewegen, immer zu derselben Wirbellinie gehörig bleiben.

3) Dafs das Product aus dem Querschnitte und der Rotationsgeschwindigkeit eines unendlich dünnen Wirbelfadens längs der ganzen Länge des Fadens constant ist, und auch bei der Fortbewegung des Fadens denselben Werth behält. Die Wirbelfäden müssen deshalb innerhalb der Flüssigkeit in sich zurücklaufen, oder können nur an ihren Grenzen endigen.

Dieser letztere Satz macht es möglich die Rotationsgeschwindigkeiten zu bestimmen, wenn die Form der betreffenden Wirbelfäden zu verschiedenen Zeiten gegeben ist. Ferner wird die Aufgabe gelöst, die Geschwindigkeiten der Wassertheilchen für einen gewissen Zeitpunkt zu bestimmen, wenn für diesen Zeitpunkt die Rolationsgeschwindigkeiten gegeben sind; nur bleibt da- 
bei eine willkührliche Function unbestimmt, welche zur Erfüllung der Grenzbedingungen verwendet werden mufs.

Diese letztere Aufgabe führt zu einer merkwürdigen Analogie der Wirbelbewegungen des Wassers mit den electromagnetischen Wirkungen electrischer Ströme. Wenn nämlich in einem einfach zusammenhängenden *), mil bewegter Flüssigkeit gefüllten Raume ein Geschwindigkeitspotential existirt, sind die Geschwindigkeiten der Wassertheilchen gleich und gleichgerichtet den Kräften, welche eine gewisse Vertheilung magnetischer Massen an der Oberfläche des Raums auf ein magnetisches Theilchen im Innern ausüben würde. Wenn dagegen in einem solchen Raume Wirbelfâden existiren,' so sind die Geschwindigkeiten der Wassertheilchen gleich zu setzen den auf ein magnetisches Theilchen ausgeübten Kräften geschlossener electrischer Ströme, welche theils durch die Wirbelfäden im Innern der Masse, theils in ihrer Oberfläche fliefsen, und deren Intensität dem Product aus dem Querschnitt der Wirbelfäden und ihrer Rotationsgeschwindigkeit proportional ist.

Ich werde mir deshalb im Folgenden öfter erlauben, die Anwesenheit von magnetischen Massen oder electrischen Strömen zu fingiren, blos um dadurch für die Natur von Functionen einen kürzeren und anschaulicheren Ausdruck zu gewinnen, die eben solche Functionen der Coordinaten sind, wie die Potentialfunctionen, oder Anziehungskräfte, welche jenen Massen oder Strömen für ein magnetisches Theilchen zukommen.

Durch diese Sätze wird die Reihe der Bewegungsformen, welche in der nicht behandelten Klasse der Integrale der hydrodynamischen Gleichungen verborgen sind, wenigstens für die Vorstellung zugänglich, wenn auch die vollständige Ausführung der Integration nur in wenigen einfachsten Fällen möglich ist, wo nur ein oder zwei geradlinige oder kreisförmige Wirbelfäden vorhanden sind in unbegrenzten oder durch eine unendliche Ebene theilweis begrenzten Wassermassen.

Es läfst sich nachweisen, dafs geradlinige parallele Wirbelfäden in einer Wassermasse, die nur durch senkrecht gegen die Fäden gestellte Ebenen be-

*) Ich nehme diesen Ausdruck in demselben Sinne, in welchem Riemann (dieses Journal Bd. LIV, S. 108) von einfach und mehrfach zusammenhängenden Flächen spricht. Ein $n$ fach zusammenhängender Raum ist danach cin solcher, durch den $n-1$, aber nicht mehrere Schnittflächen gelegt werden können, ohne den Raum in zwei vollständig getrennte Theile zu trennen. Ein Ring ist also in diesem Sinne ein zweifach zusammenhängender Raum. Die Schnittflächen müssen ringsum durch die Linie, in der sie die Oberfläche des Raums schneiden, vollständig begrenzt sein. 
grenzt ist, um ihren gemeinschaftlichen Schwerpunkt rotiren, wenn man zur Bestimmung dieses Punktes, die Rotationsgeschwindigkeit gleich der Dichtigkeit einer Masse betrachtet. Die Lage des Schwerpunkts bleibt unveränderț. Bei kreisförmigen Wirbelfäden dagegen, die alle auf einer gemeinsamen Axe senkrecht stehen, bewegt sich der Schwerpunkt ihres Querschnitts parallel der Axe fort.

S. 1.

Es sei innerhalb einer tropfbaren Flüssigkeit in dem Punkte, der durch die rechtwinkligen Coordinaten $x, y, z$ bestimmt ist, zur Zeit $t$ der Druck gleich $\boldsymbol{p}$, die den drei Coordinataxen parallelen Componenten der Geschwindigkeit $u, v, w$, die Componenten der auf die Einheit der flüssigen Masse wirkenden äufseren Kräfte $\boldsymbol{X}, \boldsymbol{Y}$ und $\boldsymbol{Z}$, und die Dichtigkeit, deren Aenderungen als verschwindend klein angesehen werden, gleich $h$, so sind die bekannten Bewegungsgleichungen für die inneren Punkte der Flüssigkeit:

$$
\left\{\begin{aligned}
X-\frac{1}{h} \cdot \frac{d p}{d x} & =\frac{d u}{d t}+u \frac{d u}{d x}+v \frac{d u}{d y}+w \frac{d u}{d z} \\
Y-\frac{1}{h} \cdot \frac{d p}{d y} & =\frac{d v}{d t}+u \frac{d v}{d x}+v \frac{d v}{d y}+w \frac{d v}{d z} \\
Z-\frac{1}{h} \cdot \frac{d p}{d z} & =\frac{d w}{d t}+u \frac{d w}{d x}+v \frac{d w}{d y}+w \frac{d w}{d z} \\
0 & =\frac{d u}{d x}+\frac{d v}{d y}+\frac{d w}{d z} .
\end{aligned}\right.
$$

Man hat bisher fast ausschliefslich nur solche Fälle behandelt, wo nicht nur die Kräfte $\boldsymbol{X}, \boldsymbol{Y}$ und $\boldsymbol{Z}$ ein Potential $\boldsymbol{V}$ haben, also auf die Form gebracht werden können

$$
\text { (1 a.) } \quad X=\frac{d V}{d x}, \quad \boldsymbol{Y}=\frac{d V}{d y}, \quad \boldsymbol{Z}=\frac{d V}{d z},
$$

sondern auch aufserdem ein Geschwindigkeitspotential $\varphi$ gefunden werden kann, so dafs

$$
\text { (1 b.) }, u=\frac{d \varphi}{d x}, \quad v=\frac{d \varphi}{d y}, \quad w=\frac{d \varphi}{d z} .
$$

Dadurch vereinfacht sich die Aufgabe aufserordentlich, indem die drei ersten der Gleichungen (1.) eine gemeinsame Integralgleichung geben, aus der $p \mathrm{zu}$ finden ist, nachdem man $\varphi$ der vierten Gleichung gemäfs bestimmt hat, welche in diesem Falle die Gestalt annimmt:

$$
\frac{d^{2} \varphi}{d x^{2}}+\frac{d^{2} \varphi}{d y^{2}}+\frac{d^{2} \varphi}{d z^{2}}=0
$$


also mit der bekannten Differentialgleichung für das Potential magnetischer Massen übereinstimmt, welche aufserhalb des Raumes liegen, für den diese Gleichung gelten soll. Auch ist bekannt, dafs jede Function $\varphi$, welche die obige Differentialgleichung innerhalb eines einfach zusammenhängenden *) Raumes erfüllt, als das Potential einer bestimmten Vertheilung magnetischer Massen an der Oberfläche des Raumes ausgedrückt werden kann, wie ich schon in der Einleitung angeführt habe.

Damit die in der Gleichung ( 1 b.) verlangte Substitution gemacht werden könne, mufs sein

$$
\text { (1 c.) } \quad \frac{d u}{d y}-\frac{d v}{d x}=0, \quad \frac{d v}{d z}-\frac{d w}{d y}=0, \quad \frac{d w}{d x}-\frac{d u}{d z}=0 .
$$

Um die mechanische Bedeutung dieser letzteren drei Bedingungen zu verstehen, können wir uns die Veränderung, welche irgend ein unendlich kleines Wasservolum in dem Zeittheilchen $d t$ erleidet, zusammengesetzt denken aus drei verschiedenen Bewegungen: 1) einer Fortführung des Wassertheilchens durch den Raum hin, 2) einer Ausdehnung oder Zusammenziehung des Theilchens nach drei Hauptdilatationsrichtungen, wobei ein jedes aus Wasser gebildete rechtwinklige Parallelepipedon, dessen Seiten den Hauptdilatationsrichtungen parallel sind, rechtwinklig bleibt, während seine Seiten zwar ihre Länge ändern, aber ihren früheren Richtungen parallel bleiben, 3) einer Drehung um eine beliehig gerichtete temporäre Rotationsaxe, welche Drehung nach einem bekannten Satze immer als Resultante dreier Drehungen um die Coordinataxen angesehen werden kann.

Sind in dem Punkte, dessen Coordinataxen $x, y$ und $z$ sind, die unter (1 c.) aufgestellten Bedingungen erfüllt, so wollen wir die Werthe von $u, v$, v und ihren Differentialquotienten in jenem Punkte folgendermafsen bezeichnen:

$$
\begin{array}{lll}
u=A, & \frac{d u}{d x}=a, & \frac{d w}{d y}=\frac{d v}{d z}=\alpha, \\
v=B, & \frac{d v}{d y}=b, & \frac{d u}{d z}=\frac{d w}{d x}=\beta, \\
w=C, & \frac{d w}{d z}=c, & \frac{d v}{d x}=\frac{d u}{d y}=\gamma
\end{array}
$$

*) In mehrfach zusammenhängenden Räumen kann $\varphi$ mehrdeutig werden, und für mehrdeutige Functionen, die der obigen Differentialgleichung Genüge thun, gilt der Fundamentalsatz von Green's Theorie der Electricität (dieses Journal Bd. XLIV, S. 360) nicht, und demgemäfs auch ein grofser Theil der aus ihm herfliefsenden Sätze nicht, welche Gau $/ s$ und Green für die magnetischen Potentialfunctionen aufgestellt haben, die ihrer Natur nach immer eindeutig sind. 
und erhalten dann für Punkte, deren Coordinaten $x, y, z$ unendlich wenig von $x, y, z$ verschieden sind:

$$
\begin{aligned}
\boldsymbol{u} & =\boldsymbol{A}+\boldsymbol{a}(\boldsymbol{x}-\mathfrak{x})+\boldsymbol{\gamma}(\boldsymbol{y}-\mathfrak{y})+\boldsymbol{\beta}(\boldsymbol{z}-\mathfrak{z}), \\
\boldsymbol{v} & =\boldsymbol{B}+\boldsymbol{\gamma}(\boldsymbol{x}-\mathfrak{x})+\boldsymbol{b}(\boldsymbol{y}-\mathfrak{y})+\boldsymbol{\alpha}(\boldsymbol{z}-\mathfrak{z}), \\
\boldsymbol{w} & =\boldsymbol{C}+\boldsymbol{\beta}(\boldsymbol{x}-\mathfrak{x})+\boldsymbol{\alpha}(\boldsymbol{y}-\mathfrak{y})+\boldsymbol{c}(\boldsymbol{z}-\mathfrak{z}),
\end{aligned}
$$

oder wenn wir setzen

$$
\begin{gathered}
\varphi=\boldsymbol{A}(\boldsymbol{x}-\mathfrak{x})+\boldsymbol{B}(y-\mathfrak{y})+\boldsymbol{C}(z-\mathfrak{z})+\frac{1}{2} \boldsymbol{a}(\boldsymbol{x}-\mathfrak{x})^{2}+\frac{1}{2} \boldsymbol{b}(y-y)^{2}+\frac{1}{2} c(z-\mathfrak{z})^{2} \\
+\alpha(y-y)(z-z)+\beta(x-x)(z-z)+\gamma(x-x)(y-y),
\end{gathered}
$$

so ist

$$
u=\frac{d \varphi}{d x}, \quad v=\frac{d \varphi}{d y}, \quad w=\frac{d \varphi}{d z} .
$$

Es ist bekannt, dafs man durch eine geeignete Wahl anders gerichteter rechtwinkliger Coordinaten $x_{1}, y_{1}, z_{1}$, deren Mittelpunkt im Punkte $x, y, z$ liegt, den Ausdruck für $\varphi$ auf die Form bringen kann

$$
\varphi=A_{1} x_{1}+B_{1} y_{1}+C_{1} z_{1}+\frac{1}{2} a_{1} x_{1}^{2}+\frac{1}{2} b_{1} y_{1}^{2}+\frac{1}{2} c_{1} z_{1}^{2},
$$

wo dann die nach diesen neuen Coordinataxen zerlegten Geschwindigkeiten $u_{1}, v_{1}, w_{1}$ die Werthe erhalten

$$
u_{1}=A_{1}+a_{1} x_{1}, \quad v_{1}=B_{1}+b_{1} y_{1}, \quad v_{1}=C_{1}+c_{1} z_{1} .
$$

Die $x_{1}$ Axe parallele Geschwindigkeit $u_{1}$ ist also gleich für alle Wassertheilchen für welche $x_{1}$ denselben Werth hat, oder Wassertheilchen, welche zu Anfang des Zeittheilchens $d t$ in einer den $y_{1} z_{1}$ parallelen Ebene liegen, sind auch am Schlusse des Zeittheilchens $d t$ in einer solchen. Dasselbe gilt für die $x_{1} y_{1}$ und $x_{1} z_{1}$ Ebene. Wenn wir also ein Parallelepipedon durch drei den letztgenannten Coordinatebenen parallele und ihnen unendlich nahe Ebenen begrenzt denken, so bilden die darin eingeschlossenen Wassertheilchen auch nach Ablauf des Zeittheilchens $d t$ ein rechtwinkliges Parallelepipedon, dessen Flächen denselben Coordinatebenen parallel sind. Die ganze Bewegung eines solchen unendlich kleinen Parallelepipedon ist also unter der in (1 c.) ausgesprochenen Voraussetzung zusammengesetzt nur aus einer Translationsbewegung im Raume, und einer Ausdehnung oder Zusammenziehung seiner Kanten, und es ist keine Drehung desselben vorhanden.

Kehren wir zurück zu dem ersten Coordinatsystem der $x, y, z$ und denken wir nun zu den bisher vorhandenen Bewegungen der den Punct $x y z$ umgebenden unendlich kleinen Wassermasse noch Rotationsbewegungen um 
Axen, die denen der $x, y$ und $z$ parallel sind, und durch den Punct xyz gehen, hinzugefügt, deren Winkelgeschwindigkeiten beziehlich sein mögen $\xi, \eta, \zeta$, so sind die davon herrührenden Geschwindigkeitscomponenten parallel den Coordinataxen der $x, y, z$ beziehlich
0 ,
$(\boldsymbol{z}-\boldsymbol{z}) \dot{\xi}$
$-(y-y) \xi$
$-(z-z) \eta$,
0 ,
$(x-x) \eta$,
$(y-y) \zeta$,
$-(x-x) \zeta$
0.

Die Geschwindigkeiten des Theilchens, dessen Coordinaten $x, y, z$ sind, werden nun also

$$
\begin{aligned}
\boldsymbol{u} & =\boldsymbol{A}+\boldsymbol{a}(\boldsymbol{x}-\mathfrak{x})+(\boldsymbol{\gamma}+\boldsymbol{\zeta})(\boldsymbol{y}-\mathfrak{y})+(\beta-\eta)(z-\mathfrak{z}), \\
\boldsymbol{v} & =\boldsymbol{B}+(\boldsymbol{\gamma}-\zeta)(x-\mathfrak{x})+\boldsymbol{b}(\boldsymbol{y}-\boldsymbol{y})+(\alpha+\xi)(z-\mathfrak{z}), \\
\boldsymbol{w} & =\boldsymbol{C}+(\beta+\eta)(\boldsymbol{x}-\mathfrak{x})+(\alpha-\xi)(y-\mathfrak{y})+\boldsymbol{c}(\boldsymbol{z}-\mathfrak{z}) .
\end{aligned}
$$

Daraus folgt durch Differenziren

$$
\text { (2.) }\left\{\begin{array}{l}
\frac{d v}{d z}-\frac{d v}{d y}=2 \xi \\
\frac{d w}{d x}-\frac{d \imath}{d z}=2 \eta \\
\frac{d u}{d y}-\frac{d v}{d x}=2 \zeta
\end{array}\right.
$$

Die Gröfsen der linken Seite also, welche nach den Gleichungen (1 c.) gleich Null sein müssen, wenn ein Geschwindigkeitspotential existiren soll, sind gleich den doppelten Rotationsgeschwindigkeiten der betreffenden Wassertheilchen um die drei Coordinataxen. Die Existenz eines Geschwindigkeitspotentials schliefst die Existenz von Rotationsbewegungen der Wassertheilchen aus.

Als eine weitere characteristische Eigenthümlichkeit der Flüssigkeitsbewegung mit einem Geschwindigkeitspotential soll hier ferner noch angeführt werden, dafs in einem ganz von festen Wänden eingeschlossenen, ganz mit Flüssigkeit gefüllten und einfach zusammenhängendem Raume $\boldsymbol{S}$ keine solche Bewegung vorkommen kann. Denn wenn wir mit $n$ die nach innen gerichtete Normale der Oberfläche eines solchen Raumes bezeichnen, mufs die zur Wand senkrecht gerichtete Geschwindigkeitscomponente $\frac{d \varphi}{d x}$ überall gleich Null sein. Dann ist nach einem bekannten Satze ${ }^{*}$ ) von Green

*) Der vorher schon angeführte Satz in diesem Journal Bd. LIV. S. 108, welcher nicht für mehrfach zusammenhä̉ngende Räume gilt. 


$$
\iint\left[\left(\frac{d \varphi}{d x}\right)^{2}+\left(\frac{d \varphi}{d y}\right)^{2}+\left(\frac{d \varphi}{d z}\right)^{2}\right] d x d y d z=-\int \varphi \frac{d \varphi}{d n} d \omega
$$

wo links die Integration über den ganzen Raum $\boldsymbol{S}$; rechts über die ganze Oberfläche von $S$, deren Flächenelement mit $d \omega$ bezeichnet ist, ausgedehnt werden mufs. Ist nun $\frac{d \varphi}{d n}$ an der ganzen Oberfläche gleich Null, so mufs auch das Integral links gleich Null sein, was nur der Fall sein kann, wenn im ganzen Raume $\boldsymbol{S}$

$$
\frac{d \varphi}{d x}=\frac{d \varphi}{d y}=\frac{d \varphi}{d z}=0,
$$

also gar keine Bewegung des Wassers stattindet. Jede Bewegung einer begrenzten Flüssigkeitsmasse in einem einfach zusammenhängenden Raume, die ein Geschwindigkeitspotential hat, ist also nothwendig mit einer Bewegung der Oberfläche der Flüssigkeit verbunden. Ist diese Bewegung der Oberfläche, d. h. $\frac{d \varphi}{d{ }^{\prime}}$ vollständig gegeben, so ist dadurch auch die ganze Bewegung der eingeschlossenen Flüssigkeitsmasse eindeutig bestimmt. Denn gäbe es zwei Funktionen $\varphi_{1}$ und $\varphi_{1}$, welche gleichzeitig im Inneren des Raumes $\boldsymbol{S}$ der Gleichung

$$
\frac{d^{2} \varphi}{d x^{2}}+\frac{d^{2} \varphi}{d y^{2}}+\frac{d^{2} \varphi}{d z^{2}}=0
$$

und an der Oberfläche die Bedingung

$$
\frac{d \varphi}{d n}=\psi
$$

erfüllten, wo $\psi$ die durch die gegebene Bewegung der Oberfläche bedingten Werthe von $\frac{d \varphi}{d n}$ bezeichnet, so würde auch die Function $\left(\varphi_{1}-\varphi_{1}\right)$ die èrstere Bedingung im Innern von $\boldsymbol{S}$ erfüllen, an der Oberfläche aber

$$
\frac{d\left(\varphi_{1}-\varphi_{11}\right)}{d n}=0
$$

sein, woraus wie eben gezeigt ist, auch für das ganze Innere von $\boldsymbol{S}$ folgen würde

$$
\frac{d\left(\varphi_{1}-\varphi_{11}\right)}{d x}=\frac{d\left(\varphi_{1}-\varphi_{11}\right)}{d y}=\frac{d\left(\varphi_{1}-\varphi_{11}\right)}{d z}=0 .
$$

Beiden Functionen würdẹn also genau dieselben Geschwindigkeiten auch im ganzen Innern von $\boldsymbol{S}$ entsprechen.

Also nur in dem Falle, wo kein Geschwindigkeitspotential existirt, können Drehungen der Wassertheilchen, und in sich zurücklaufende Bewegungen 
innerhalb einfach zusammenhängender ganz geschlossener Räume vorkommen. Wir können daher die Bewegungen, denen ein Geschwindigkeitspotential nicht zukommt, im Allgemeinen als Wirbelbewegungen characterisiren.

\section{\$. 2.}

Wir wollen zunächst die Aenderungen der Rotationsgeschwindigkeiten $\xi$, $\eta$ und $\zeta$ während der Bewegung bestimmen, wenn nur Kräfte wirken, denen ein Kräftepotential zukommt. Ich bemerke zunächst im Allgemeinen, dafs wenn $\psi$ eine Function von $x, y, z, t$ ist, und um $\partial \psi$ wächst, während die letzteren vier Gröfsen um $\partial x, \partial y, \partial z$ und $\partial t$ wachsen, wir haben

$$
\partial \psi=\frac{d \psi}{d t} \partial t+\frac{d \psi}{d x} \partial x+\frac{d \psi}{d y} \partial y+\frac{d \psi}{d z} \partial z \text {. }
$$

Soll nun die Aenderung von $\psi$ während des Zeittheilchens $\partial t$ für ein constant bleibendes Wassertheilchen bestimmt werden, so mũssen wir den Grōfsen $\partial x, \partial y$ und $\partial z$ dieselben Werthe geben, welche sie für das bewegte Wassertheilchen haben, nämlich

$$
\partial x=u \partial t, \quad \partial y=v \partial t, \quad \partial z=w \partial t
$$

und erhalten

$$
\frac{\partial \psi}{\partial t}=\frac{d \psi}{d t}+u \frac{d \psi}{d x}+v \frac{d \psi}{d y}+w \frac{d \psi}{d z}
$$

Das Zeichen $\frac{\partial \psi}{\partial t}$ werde ich im Folgenden immer nur in dem Sinne gebrauchen, dafs $\frac{\partial \psi}{\partial t} d t$ die Aenderung von $\psi$ während der Zeit $d t$ für dasselbe Wassertheilchen bezeichnet, dessen Coordinaten zu Anfang der Zeit $d t x, y$ und $z$ waren.

Indem wir aus den ersten der Gleichungen (1.) mit Hülfe von Differentiationen die Gröfse $p$ eliminiren, und dabei die Bezeichnungen der Gleichungen (2.) einführen, und für die Kräfte $X, Y, Z$ die Gleichungen (1 a.) als erfüllbar betrachten, erhalten wir folgende drei Gleichungen:

oder auch

$$
\left\{\begin{array}{l}
\frac{\partial \xi}{\partial t}=\xi \frac{d u}{d x}+\eta \frac{d u}{d y}+\zeta \frac{d u}{d z} \\
\frac{\partial \eta}{\partial t}=\xi \frac{d v}{d x}+\eta \frac{d v}{d y}+\xi \frac{d v}{d z} \\
\frac{\partial \zeta}{\partial t}=\xi \frac{d w}{d x}+\eta \frac{d w}{d y}+\zeta \frac{d w}{d z}
\end{array}\right.
$$




$$
\text { (3 a.) }\left\{\begin{array}{l}
\frac{\partial \xi}{\partial t}=\xi \frac{d u}{d x}+\eta \frac{d v}{d x}+\zeta \frac{d w}{d x}, \\
\frac{\partial \eta}{\partial t}=\xi \frac{d u}{d y}+\eta \frac{d v}{d y}+\zeta \frac{d w}{d y}, \\
\frac{\partial \zeta}{\partial t}=\xi \frac{d u}{d z}+\eta \frac{d v}{d z}+\zeta \frac{d w}{d z} .
\end{array}\right.
$$

Wenn in einem Wassertheilchen $\xi, \eta$ und $\zeta$ gleichzeitig gleich Null sind, sind auch

$$
\frac{\partial \xi}{\partial t}=\frac{\partial \eta}{\partial t}=\frac{\partial \zeta}{\partial t}=0
$$

Diejenigen Wassertheilchen also, welche nicht schon Rotationsbewegungen haben, bekommen auch im Verlaufe der Zeit keine Rotationsbewegungen.

Bekanntlich kann man Rotationen nach der Methode des Parallelogramms der Kräfte zusammensetzen. Sind $\xi, \eta, \zeta$ die Rotationsgeschwindigkeiten um die Coordinataxen, so ist die Rotationsgeschwindigkeit $q$ um die augenblickliche Axe der Rotation

$$
\boldsymbol{q}=\sqrt{\xi^{2}+\eta^{2}+\zeta^{2}}
$$

und die Cosinus der Winkel, welche diese Axe mit den Coordinaten bildet, sind beziehlich $\frac{\xi}{q}, \frac{\eta}{q}$ und $\frac{\zeta}{q}$.

Wenn wir nun in Richtung dieser augenblicklichen Drehungsaxe, das unendlich kleine Stück $q \varepsilon$ abschneiden, so sind die Projectionen dieses Stückes auf die drei Coordinataxen beziehlich $\varepsilon \xi$, $\varepsilon \eta$ und $\varepsilon \xi$. Während im Punkte $x, y, z$ die Componenten der Geschwindigkeit $u, v$ und $w$ sind, sind sie am anderen Endpunkt von $q \varepsilon$ beziehlich

$$
\begin{aligned}
& u_{1}=u+\varepsilon \xi \frac{d u}{d x}+\varepsilon \eta \frac{d u}{d y}+\varepsilon \zeta \frac{d u}{d z} \\
& v_{1}=v+\varepsilon \xi \frac{d v}{d x}+\varepsilon \eta \frac{d v}{d y}+\varepsilon \zeta \frac{d v}{d z} \\
& w_{1}=w+\varepsilon \xi \frac{d w}{d x}+\varepsilon \eta \frac{d w}{d y}+\varepsilon \zeta \frac{d w}{d z}
\end{aligned}
$$

Nach Verlauf der Zeit dt haben also die Projectionen der Entfernung der beiden Wassertheilchen, welche zu Anfang von $d t$ das Stück $q \varepsilon$ begrenzten, einen Werth erlangt, welchen man mit Berücksichtigung der Gleichungen (3.) folgendermafsen schreiben kann: 
3. Helmholtz, über Integrale der hydrodynamischen Gleichungen.

$$
\begin{aligned}
\varepsilon \xi+\left(u_{1}-u\right) d t & =\varepsilon\left(\xi+\frac{\partial \xi}{\partial t} d t\right) \\
\varepsilon \eta+\left(v_{1}-v\right) d t & =\varepsilon\left(\eta+\frac{\partial \eta}{\partial t} d t\right) \\
\varepsilon \zeta+\left(w_{1}-v\right) d t & =\varepsilon\left(\zeta+\frac{\partial \zeta}{\partial t} d t\right) .
\end{aligned}
$$

Links stehen hier die Projectionen der neuen Lage der Verbindungslinie $q \varepsilon$, rechts die mit dem constanten Factor $\varepsilon$ multiplicirten Projectionen der neuen Rotationsgeschwindigkeit; es folgt aus diesen Gleichungen, dafs die -Verbindungslinie der beiden Wassertheilchen, welche zu Anfang der Zeit dt das Stück $q \varepsilon$ der augenblicklichen Rotationsaxe begrenzten, auch nach Ablauf der Zeit $d t$ noch mit der jetzt geänderten Rotationsaxe zusammenfällt.

Wenn wir eine Linie, deren Richtung überall mit der Richtung der augenblicklichen Rotationsaxe der dort befindlichen Wassertheilchen zusammentrifft, wie oben festgesetzt ist, eine Wirbellinie nennen, so können wir den eben gefundenen Satz so aussprechen: Eine jede Wirbellinie bleibt fortdauernd aus denselben Wassertheilchen zusammengesetzt, während sie mit diesen Wassertheilchen in der Flüssigkeit fortschwimmt.

Die rechtwinkligen Componenten der Rotationsgeschwindigkeit nehmen in demselben Verhältnisse zu, wie die Projectionen des Stücks $\varepsilon q$ der Rotationsaxe; daraus folgt, dafs die Grö/se der resultircnden liotationsgeschwindigkeit in einem bestimmten Wassertheilchen in demselben Verhültnisse sich veründert, wie der Abstand dieses Wassertheilchens von seinen Nachbarn in der Rotationsaxe.

Denken wir uns durch alle Punkte des Umfangs einer unendlich kleinen Fläche Wirbellinien gelegt, so wird dadurch aus der Flüssigkeit ein Faden von unendlich kleinem Querschnitt herausgetheilt, der Wirbelfaden genannt werden soll. Das Volumen eines zwischen zwei bestimmten Wassertheilchen gelegenen Stücks eines solchen Fadens, welches nach den eben bewiesenen Sätzen immer von denselben Wassertheilchen angefüllt bleibt, mufs bei der Fortbewegung constant bleiben, sein Querschnitt sich also im umgekehrten Verhältnisse als die Länge ändern. Danach kann man den eben hingestellten Satz auch so aussprechen: Das Product aus der Rotationsgeschwindigkeit und dem Querschnitt in einem aus denselben Wassertheilchen bestehenden Stitcke eines Wirbelfadens bleibt bei der Fortbewegung desselben constant. 
3. Helmholtz, über Integrale der hydrodynamischen Gleichungen.

Aus den Gleichungen (2.) folgt unmittelbar, dafs

Daraus weiter, dafs

$$
\frac{d \xi}{d x}+\frac{d \eta}{d y}+\frac{d \zeta}{d z}=0 \text {. }
$$

$$
\iiint\left(\frac{d \xi}{d x}+\frac{i d \eta}{d y}+\frac{d \zeta}{d z}\right) d x d y d z=0
$$

wobei die Integration über einen ganz beliebigen Theil $\boldsymbol{S}$ der Wassermasse ausgedehnt werden kann. Wenn wir partiell integriren, folgt daraus

$$
\iint \xi d y d z+\iint \eta d x d z+\iint \zeta d x d y=0,
$$

wobei die Integrationen über die ganze Oberfläche des Raumes $\boldsymbol{S}$ auszudehnen sind. Nennen wir $d \omega$ ein Flächenelement dieser Oberfläche und $\alpha, \beta, \gamma$ die drei Winkel, welche die nach aufsen gerichtete Normale von $d \omega$ mit den Coordinataxen bildet, so ist

$$
d y d z=\cos \alpha d \omega, \quad d x d z=\cos \beta d \omega, \quad d x d y=\cos \gamma d \omega,
$$

also

$$
\iint(\xi \cos \alpha+\eta \cos \beta+\zeta \cos \gamma) d \omega=0,
$$

oder wenn man $q$ die resultirende Rotationsgeschwindigkeit nennt, und $\vartheta$ den Winkel zwischen ihr und der Normale,

$$
\iint q \cos \vartheta \cdot d \omega=0
$$

die Integration über die ganze Oberfläche von $\boldsymbol{S}$ ausgedehnt.

Nun sei $\mathbf{S}$ ein Stück eines Wirbelfadens, begrenzt durch zwei unendlich kleine senkrecht gegen die Axe des Fadens gelegte Ebenen $\omega_{1}$ und $\omega_{\text {", }}$, so ist $\cos \vartheta$ an einer dieser Ebenen gleich 1, an der andern -1 , an der ganzen übrigen Oberfläche des Fadens gleich 0 , folglich wenn $q_{\text {, und }} q_{11}$ die Rotationsgeschwindigkeiten in $\omega_{\text {, }}$ und $\omega_{\text {" }}$ sind, reducirt sich die letzte Gleichung auf

$q_{1} \omega_{1}=q_{11} \omega_{11}$,
woraus folgt: Das Product aus der Rotationsgeschwindigkeit und dem Querschnitt ist in der ganzen Länge desselben Wirbelfadens constant. Dafs es sich auch bei der Fortbewegung des Fadens nicht ändert, ist vorher schon bewiesen worden.

Es folgt hieraus auch, dafs ein Wirbelfaden nirgends innerhalb der Flüssigkeit aufhören dürfe, sondern entweder ringförmig innerhglb der Flüssigkeit in sich zurücklaufen, oder bis an die Grenzen der Flüssigkeit reichen 
müsse. Denn wenn ein Wirbelfaden innerhalb der Flüssigkeit irgend wo endete, würde sich eine geschlossene Fläche construiren lassen, für welche das Integral $\int q \cos \vartheta d \omega$ nicht den Werth Null hätte.

\section{S. 3.}

Wenn man die Bewegung der in der Flüssigkeit vorhandenen Wirbelfäden bestimmen kann, so werden durch die hingestellten Sätze auch die Gröfsen $\xi, \eta$ und $\zeta$ vollständig zu bestimmen sein. Wir wollen jetzt an die Aufgabe gehen, aus den Gröfsen $\xi$, $\eta$ und $\zeta$ die Geschwindigkeiten $u$, $v$ und $\boldsymbol{w}$ zu finden.

Es seien also innerhalb einer Wassermasse, die den Raum $\boldsymbol{S}$ einnimmt, die Werthe von $\xi, \eta$ und $\zeta$ gegeben, welche drei Gröfsen der Bedingung genūgen, dafs

$$
\text { (2 a.) } \frac{d \xi}{d x}+\frac{d \eta}{d y}+\frac{d \zeta}{d z}=0 \text {. }
$$

Es sollen gefunden werden $u, v$ und $w$, so dafs sie innechalb des ganzen Raumes $\boldsymbol{S}$ den Bedingungen genügen, dafs

$$
\begin{aligned}
& \text { (1.) } \frac{d u}{d x}+\frac{d v}{d y}+\frac{d w}{d z}=0 \\
& \text { (2.) }\left\{\begin{array}{l}
\frac{d v}{d z}-\frac{d w}{d y}=2 \xi, \\
\frac{d w}{d x}-\frac{d u}{d z}=2 \eta, \\
\frac{d u}{d y}-\frac{d v}{d x}=2 \xi .
\end{array}\right.
\end{aligned}
$$

Dazu kommen noch die durch die jedesmalige Natur der Aufgabe für die Grenze des Raumes $\boldsymbol{S}$ geforderten Bedingungen.

Bei der gegebenen Vertheilung von $\xi, \eta, \zeta$ können nun theils Wirbellinien vorkommen, welche innerhalb des Raumes $\boldsymbol{S}$ geschlossen in sich zurücklaufen, theils solche welche die Grenze von $\boldsymbol{S}$ erreichen und hier abbrechen. Wenn letzteres der Fall ist, so kann man jedenfalls entweder auf der Oberfläche von $\boldsymbol{S}$ oder aufserhalb $\boldsymbol{S}$ diese Wirbellinien fortsetzen und in sich zurücklaufend schliefsen, so dafs dann ein gröfserer Raum $\boldsymbol{S}_{1}$ existirt, welcher nur geschlossene Wirbellinien enthält, und an dessen ganzer Oberfläche $\xi, \eta, \zeta$ und ihre Resultante $q$ selbst gleich Null sind, oder wenigstens

(2b.) $\xi \cos \alpha+\eta \cos \beta+\zeta \cos \gamma=q \cos \vartheta=0$. 
Wie vorher bedeuten hier $\alpha, \beta, \gamma$ die Winkel zwischen der Normale des betreffenden Theils der Oberfläche von $\boldsymbol{S}_{1}$ und den Coordinataxen, $\vartheta$ den Winkel zwischen der Normale und der resultirenden Rotationsaxe.

Werthe von $u, v, w$, welche den Gleichungen (1.) $)_{4}$ und (2.) genügen, erhalten wir nun, indem wir setzen

$$
\text { (4.) }\left\{\begin{array}{l}
u=\frac{d P}{d x}+\frac{d N}{d y}-\frac{d M}{d z} \\
v=\frac{d P}{d y}+\frac{d L}{d z}-\frac{d N}{d x} \\
w=\frac{d P}{d z}+\frac{d M}{d x}-\frac{d L}{d y}
\end{array}\right.
$$

und die Gröfsen $\boldsymbol{L}, \boldsymbol{M}, \boldsymbol{N}, \boldsymbol{P}$ durch die Bedingungen bestimmen, dafs innerhalb des Raumes $\boldsymbol{S}_{1}$

$$
\text { (5.) }\left\{\begin{array}{l}
\frac{d^{2} L}{d x^{2}}+\frac{d^{2} L}{d y^{2}}+\frac{d^{2} L}{d z^{2}}=2 \xi \\
\frac{d^{2} M}{d x^{2}}+\frac{d^{2} M}{d y^{2}}+\frac{d^{2} M}{d z^{2}}=2 \eta \\
\frac{d^{2} N}{d x^{2}}+\frac{d^{2} N}{d y^{2}}+\frac{d^{2} N}{d z^{2}}=2 \zeta, \\
\frac{d^{2} P}{d x^{2}}+\frac{d^{2} P}{d y^{2}}+\frac{d^{2} P}{d z^{2}}=0 .
\end{array}\right.
$$

Wie diese letzteren Gleichungen integrirt werden, ist bekannt. $\boldsymbol{L}, \boldsymbol{M}, \boldsymbol{N}$ sind die Potentialfunctionen fingirter magnetischer Massen, die mit der Dichtigkeit $-\frac{\xi}{2 \pi},-\frac{\eta}{2 \pi}$ und $-\frac{\zeta}{2 \pi}$ durch den Raum $\boldsymbol{S}_{1}$ verbreitet sind, $\boldsymbol{P}$ die Potentialfunction von Massen, die aufserhalb des Raumes $\boldsymbol{S}$ liegen. Bézeichnen wir die Entfernung eines Punktes, dessen Coordinaten $a, \dot{b}, c$ sind, von dem Punkte $x, y, z$ mit $r$, und mit $\xi_{a}, \eta_{a}, \zeta_{a}$ die Werthe von $\xi, \eta$, $\zeta$ in dem Punkte $a, b$, so ist also

$$
\text { (5 a.) }\left\{\begin{array}{l}
\boldsymbol{L}=-\frac{1}{2 \pi} \iiint \frac{\xi_{a}}{r} d a d b d c \\
M=-\frac{1}{2 \pi} \iiint \frac{\eta_{a}}{r} d a d b d c \\
N=-\frac{1}{2 \pi} \iiint \frac{\zeta_{a}}{r} d a d b d c,
\end{array}\right.
$$

die Integrationen über den Raum $\boldsymbol{S}_{1}$ ausgedehnt, und

$$
\boldsymbol{P}=\iiint \frac{k}{r} d a d b d \dot{c},
$$


wo $k$ eine willkührliche Function von $a, b, c$ ist, und die Integration über den äufseren, $\boldsymbol{S}$ umschliefsenden Raum auszudehnen ist. Die willkührliche Function $k$ mufs so bestimmt werden, dafs die Grenzbedingungen erfüllt werden, eine Aufgabe, deren Schwierigkeit ähnlich denen über electrische und magnetische Vertheilung ist.

Dafs die in (4.) gegebenen Werthe von $u, v$ und $w$ die Bedingung (1.) ${ }_{4}$ erfüllen, ergiebt sich gleich durch Differentiation mit Berücksichtigung der vierten der Gleichungen (5.).

Ferner findet man durch Differentiation der Gleichungen (4.) mit Berücksichtigung der ersten drei von (5.), dafs

$$
\begin{aligned}
& \frac{d v}{d z}-\frac{d w}{d y}=-2 \xi-\frac{d}{d x}\left[\frac{d L}{d x}+\frac{d M}{d y}+\frac{d \dot{N}}{d z}\right] \\
& \frac{d w}{d x}-\frac{d u}{d z}=2 \eta-\frac{d}{d y}\left[\frac{d L}{d x}+\frac{d M}{d y}+\frac{d N}{d z}\right] \\
& \frac{d u}{d y}-\frac{d v}{d x}=2 \zeta-\frac{d}{d z}\left[\frac{d L}{d x}+\frac{d M}{d y}+\frac{d N}{d z}\right] .
\end{aligned}
$$

Die Gleichungen (2.) sind also ebenfalls erfüllt, wenn nachgewiesen werden kann, dafs im ganzen Raume $\boldsymbol{S}_{\mathbf{1}}$

$$
\text { (5 b.) } \frac{d L}{d x}+\frac{d M}{d y}+\frac{d N}{d z}=0 \text {. }
$$

Dafs dies der Fall sei, ergiebt sich aus den Gleichungen (5 a.)

$$
\frac{d L}{d x}=+\frac{1}{2 \pi} \iiint \frac{\xi_{a}(x-a)}{r^{3}} d a d b d c,
$$

oder nach partieller Integration

$$
\begin{aligned}
& \frac{d L}{d x}=\frac{1}{2 \pi} \iint \frac{\xi_{a}}{r} d b d c-\frac{1}{2 \pi} \iiint \frac{1}{r} \cdot \frac{d \xi_{a}}{d a} d a d b d c, \\
& \frac{d M}{d y}=\frac{1}{2 \pi} \iint \frac{\eta_{a}}{r} d a d c-\frac{1}{2 \pi} \iiint \frac{1}{r} \cdot \frac{d \eta_{a}}{d b} d a d b d c, \\
& \frac{d N}{d z}=\frac{1}{2 \pi} \iint \frac{\zeta_{a}}{r} d a d b-\frac{1}{2 \pi} \iiint \frac{1}{r} \cdot \frac{d \zeta_{a}}{d c} d a d b d c .
\end{aligned}
$$

Addiren wir diese drei Gleichungen und nennen das Flächenelement der Oberfläche von $\boldsymbol{S}$ wieder $d \omega$, so erhalten wir

$$
\begin{aligned}
\frac{d L}{d x}+\frac{d M}{d y}+\frac{d N}{d z}=\frac{1}{2 \pi} \int\left(\xi_{a} \cos \alpha\right. & \left.+\eta_{a} \cos \beta+\zeta_{a} \cos \gamma\right) \frac{1}{r} d \omega \\
& -\frac{1}{2 \pi} \iiint \frac{1}{r}\left(\frac{d \xi_{a}}{d a}+\frac{d \eta_{a}}{d b}+\frac{d \zeta_{a}}{d c}\right) d a d b d c .
\end{aligned}
$$


Da aber im ganzen Innern des Raumes

$$
\text { (2 a.) } \frac{d \xi_{a}}{d a}+\frac{d \eta_{a}}{d b}+\frac{d \zeta_{a}}{d c}=0
$$

und auf seiner ganzen Oberfläche

$$
\text { (2 b.) } \quad \xi_{a} \cos \alpha+\eta_{a} \cos \beta+\zeta_{a} \cos \gamma=0,
$$

so sind beide Integrale gleich 0 und die Gleichung $(5 \mathrm{~b}$.) wie die Gleichungen (2.) erfüllt. Die Gleichungen (4.) und (5.) oder (5 a.) sind somit wirklich Integrale der Gleichungen (1.) ${ }_{4}$ und (2.)

Die in der Einleitung erwähnte Analogie zwischen den Fernwirkungen der Wirbelfäden und den electromagnetischen Fernwirkungen stromleitender Dräthe, welche ein sehr gutes Mittel abgiebt, um die Form der Wirbelbewegungen anschaulich zu machen, ergiebt sich aus diesen Sätzen.

Wenn wir die Werthe von $\boldsymbol{L}, \boldsymbol{M}, \boldsymbol{N}$ aus den Gleichungen (5 a.) in die Gleichung (4.) setzen, und diejenigen unendlich kleinen Theile von $u, v$ und $w$, welche in den Integralen von dem Körperelement $d a, d b, d c$ herrühren, mit $\Delta u, \Delta v, \Delta w$ bezeichnen, ihre Resultante mit $\Delta p$, so ist

$$
\begin{aligned}
& \Delta u=\frac{1}{2 \pi} \frac{(y-b) \zeta_{a}-(z-c) \eta_{a}}{r^{3}} d a d b d c \\
& \Delta v=\frac{1}{2 \pi} \frac{(z-c) \xi_{a}-(x-a) \xi_{a}}{r^{3}} d a d b d c \\
& \Delta w=\frac{1}{2 \pi} \frac{(x-a) \eta_{a}-(y-b) \xi_{a}}{r^{3}} d a d b d c
\end{aligned}
$$

aus diesen Gleichungen geht hervor, dafs

$$
\Delta u(x-a)+\Delta v(y-b)+\Delta w(z-c)=0,
$$

d. h. die Resultante $\Delta p$ von $\Delta u, \Delta v$ und $\Delta w$ macht mit $r$ einen rechten Winkel. Ferner

$$
\xi_{a} \Delta u+\eta_{a} \Delta v+\zeta_{a} \Delta w=0
$$

d. h. dieselbe Resultante $\Delta p$ macht auch mit der resultirenden Rotationsaxe in $a, b, c$ einen rechten Winkel. Endlich.

$$
\Delta p=\sqrt{\Delta u^{2}+\Delta v^{2}+\Delta w^{2}}=\frac{d a d b d c}{2 \pi r^{2}} q \sin v,
$$

wo $q$ die Resultante von $\xi_{a}, \eta_{a}, \zeta_{a}$ und $\nu$ der Winkel zwischen ihr und $r$ ist, welcher durch die Gleichung bestimmt wird

$$
\text { gr } \cos \nu=(x-a) \xi_{a}+(y-b) \eta_{a}+(z-c) \zeta_{a} \text {. }
$$


5. Helmholtz, über Integrale der hydrodynamischen Gleichungen.

Jedes rotirende Wassertheilchen a bedingt also in jedem anderen Theilchen $b$ derselben Wassermasse eine Geschwindigkeit, welche senkrecht gegen die durch die Rotationsaxe von a und das Theilchen b gelegte Ebene stelt. Die Grö/se dieser Geschwindigkeit ist direct proportional dem Volumen von a, seiner Rotationsgeschuindigkeit und dem Sinus des Winkels zuischen der Linie ab und der Rotationsaxe, ungekehrt proportional dem Quadrat der Entfernung beider. Theilchen.

Genau demselben Gesetze folgt die Kraft, welche eine in $a$ befindliche electrische, der Rotationsaxe parallele Strömung auf ein in $\boldsymbol{b}$ befindliches magnetisches Theilchen ausüben würde.

Die mathematische Verwandtschaft beider Klassen von Naturerscheinungen beruht darin, dafs bei den Wasserwirbeln in denjenigen Theilen der Wassermasse, welche keine Rotation haben, ein Geschwindigkeitspotential $\varphi$ existirt, welches der Gleichung

$$
\frac{d^{2} \varphi}{d x^{2}}+\frac{d^{2} \varphi}{d y^{2}}+\frac{d^{2} \varphi}{d z^{2}}=0
$$

Genüge thut, welche Gleichung nur innerhalb der Wirbelfäden nicht gilt. Wenn wir uns die Wirbelfäden aber immer als geschlossen denken entweder innerhalb oder aufserhalb der Wassermasse, so ist der Raum, in welchem die Differentialgleichung für $\varphi$ gilt, ein mehrfach zusammenhängender, denn er bleibt noch zusammenhängend, wenn man Schnittflächen durch ihn gelegt denkt, deren jede durch einen Wirbelfaden vollständig begrenzt wird. In šlchen mehrfach zusammenhängenden Räumen kann nun eine Function $\varphi$, welche der obigen Differentiaigleichung genügt, mehrdeutig werden, und sie mufs mehrdeutig werden, wenn sie in sich selbst zurücklaufende Strömungen darstellen soll, denn da die Geschwindigkeiten der Wassermasse aufserhalb der Wirbelfäden den Differentialquotienten von $\varphi$ proportional sind, so mufs man der Bewegung des Wassers folgend zu immer gröfseren Werthen von $\varphi$ fortschreiten. Ist die Strömung also in sich zurūcklaufend, und kommt man ihr folgend schliefslich an den Ort zurück, wo man schon früher war, so findet man für diesen einen zweiten höheren Werth von $\varphi$. Da man dasselbe unendlich oft ausführen kann, so mufs es unendlich viel verschiedene Werthe von $\varphi$ für jeden Punkt eines solchen mehrfach zusammenhängenden Raumes geben, welche um gleiche Differenzen von einander verschieden sind, wie die verschiedenen Werthe von $\operatorname{Arctang}\left(\frac{x}{y}\right)$, welches eine solche mehrdeutige Function ist, die der obigen Differentialgleichung genügt. 
Ebenso verhält es sich mit den electromagnetischen Wirkungen eines geschlossenen electrischen Stromes. Derselbe wirkt in die Ferne, wie eine gewisse Vertheilung magnetischer Massen auf einer von dem Stromleiter begrenzten Fläche. Aufserhalb des Stromes können deshalb die Kräfte, die er auf ein magnetisches Theilchen ausübt, als die Differentialquotienten einer Potentialfunction $\boldsymbol{V}$ betrachtet werden, welche der Gleichung genügt

$$
\frac{d^{2} V}{d x^{2}}+\frac{d^{2} V}{d y^{2}}+\frac{d^{2} V}{d z^{2}}=0 \text {. }
$$

Auch hier ist aber der Raum, welcher den geschlossenen Stromleiter umgiebt, und in dem diese Gleichung gilt, mehrfach zusammenhängend, und $\boldsymbol{V}$ vieldeutig.

Bei den Wirbelbewegungen des Wassers also, wie bei den electromagnetischen Wirkungen, hängen Geschwindigkeiten oder Kräfte aufserhalb des von Wirbelfäden oder electrischen Strömen durchzogenen Raumes von mehrdeutigen Potentialfunctionen ab, welche übrigens der allgemeinen Differentialgleichung der magnetischen Potentialfunctionen Genüge thun, während innerhalb des von Wirbelfäden oder electrischen Strömen durchzogenen Raumes statt der Potentialfunctionen, die hier nicht existiren, andere gemeinsame Functionen auftreten, wie sie in den Gleichungen (4.), (5.) und (5 a.) hingestellt sind. Bei den einfach fortströmenden Wasserbewegungen und den magnetischen Kräften dagegen haben wir es mit eindeutigen Potentialfunctionen zu thun, ebenso wie bei der Gravitation, den electrischen Anziehungskräften, den constant gewordenen electrischen und thermischen Strömungen.

Diejenigen Integrale der hydrodynamischen Gleichungen, in denen ein eindeutiges Geschwindigkeitspotential existirt, können wir Integrale erster Klasse nennen. Diejenigen dagegen, bei welchen Rotationen eines Theils der Wassertheilchen und demgemäfs in den nicht rotirenden Wassertheilchen ein mehrdeutiges Geschwindigkeitspotential vorkommt, Integrale zweiter Klasse. Es kann vorkommen, dafs im letzteren Falle nur solche Theile des Raumes in der Aufgabe zu betrachten sind, welche keine rotirenden Wassertheile enthalten, z. B. bei Bewegungen des Wassers in ringförmigen Gefäfsen, wobei ein Wirbelfaden durch die Axe des Gefäfses gehend gedacht werden kann, und wo also die Aufgabe doch noch zu denen gehört, die mittelst der Annahme eines Geschwindigkeitspotentials gelöst werden können.

In den hydrodynamischen Integralen erster Klasse sind die Geschwindigkeiten der Wassertheilchen gleich gerichtet und proportional den Kräften, welche eine gewisse aufserhalb der Flüssigkeit befindliche Vertheilung magne- 
tischer Massen auf ein am Orte des Wassertheilchens befindliches magnetisches Theilchen hervorbringen würde.

In den hydrodynamischen Integralen zweiter Klasse sind die Geschwindigkeiten der Wassertheilchen gleich gerichtet und proportional den auf ein magnetisches Theilchen wirkenden Kräften, welche geschlossene electrische, durch die Wirbelfäden fliefsende Ströme, deren Dichtigkeit der Rotationsgeschwindigkeit dieser Fäden proportional wäre, vereint mit aufserhalb der Flüssigkeit befindlichen magnetischen Massen hervorbringen würden. Die electrischen Ströme innerhalb der Flüssigkeit würden mit dem betreffenden Wirbelfaden fortfliefsen und constante Intensität behalten müssen. Die angenommene Vertheilung magnetischer Massen aufserhalb der Flüssigkeit oder auf ihrer Oberfläche mufs so bestimmt werden, dafs den Grenzbedingungen Genüge geschieht. Jede magnetische Masse kann bekanntlich auch durch electrische Strömungen ersetzt werden. Statt also in den Werthen für $u, v$ und $w$ noch die Potentialfunction $\boldsymbol{P}$ einer aufserhalb liegenden Masse $\%$ hinzuzufügen, erhält man eine ebenso allgemeine Lösung, wenn man den Gröfsen $\xi, \eta$ und $\zeta$ aufserhalb oder selbst nur an der Oberfläche der Flüssigkeit beliebige Werthe ertheilt, aber so, dafs nur geschlossene Stromfäden entstehen, und dann die Integration in den Gleichungen ( 5 a.) über den ganzen Raum ausdehnt, in welchem $\xi, \eta$ und $\zeta$ von 0 verschieden sind.

\$. 4.

In den hydrodynamischen Integralen erster Art genügt es, wie ich oben gezeigt habe, die Bewegung der Oberfläche zu kennen. Dadurch ist die Bewegung im Innern der Flüssigkeit ganz bestimmt. Bei den Integralen zweiter Art ist dagegen noch die Bewegung der innerhalb der Flüssigkeit befindlichen Wirbelfäden unter ihrem gegenseitigen Einflusse und mit Berücksichtigung der Grenzbedingungen zu bestimmen, wodurch die Aufgabe viel verwickelter wird. Indessen läfst sich für gewisse einfache Fälle auch diese Aufgabe lösen, namentlich für solche, wo Rotation der Wassertheilchen nur in gewissen Flächen oder Linien vorkommt, und die Gestalt dieser Flächen und Linien bei der Fortbewegung unverändert bleibt.

Die Eigenschaften von Flächen, welchen eine unendlich dünne Schicht rotirender Wassertheilchen anliegt, ergeben sich leicht aus den Gleichungen (5 a.). Wenn $\xi, \eta$ und $\zeta$ nur in einer unendlich dünnen Schicht von 0 verschieden sind, so werden ihre Potentialfunctionen $\boldsymbol{L}, \boldsymbol{M}$ und $\boldsymbol{N}$ nach bekannten Sätzen auf beiden Seiten der Schicht gleiche Werthe haben, aber 
ihre Differentialquotienten, in Richtung der Normale der Schicht genommen, werden verschieden sein. Denken wir uns die Coordinataxen so gelegt, dafs an der von uns betrachteten Stelle der Wirbelfläche die $\approx$ Axe der Normale der Fläche, die $x$ Axe der Rotationsaxe der Wassertheilchen in der Fläche entspricht, so dafs an dieser Stelle $\eta=\zeta=0$, so werden die Potentiale $\boldsymbol{M}$ und $\boldsymbol{N}$, so wie ihre Differentialquotienten auf beiden Seiten der Schicht dieselben Werthe haben, eben so $L$ und $\frac{d L}{d x}$ und $\frac{d L}{d y}$, dagegen wird $\frac{d L}{d z}$ zwei verschiedene Werthe haben, deren Unterschied gleich $2 \xi \varepsilon$ ist, wenn $\varepsilon$ die Dicke der Schicht bezeichnet. Demgemäfs ergeben die Gleichungen (4.), dafs $u$ und $w$ auf beiden Seiten der Wirbelfläche gleiche Werthe haben, $v$ aber Werthe, die um $2 \xi \varepsilon$ von einander verschieden sind. Es ist also auf beiden Seiten einer Wirbelfläche diejenige Componente der Geschwindigkeit, welche senkrecht gegen die Wirbellinien stehend die Fläche tangirt, von verschiedenem Werthe. Innerhalb der Schicht rotirender Wassertheilchen mufs man sich die betreffende Componente der Geschwindigkeit gleichmäfsig zunehmend denken von demjenigen Werthe, der an der einen Seite der Fläche stattfindet, zu dem der andern Seite. Denn wenn $\xi$ durch die ganze Dicke der Schicht hier constant ist, und $\alpha$ einen ächten Bruch bezeichnet, $v^{\prime}$ den Werth von $v$ auf der einen, $v_{1}$ auf der andern Seite, $v_{\alpha}$ in der Schicht selbst um $\alpha \varepsilon$ von der ersten Seite entfernt, so sahen wir, dafs $v^{\prime}-v_{1}=2 \xi \varepsilon$, weil zwischen beiden eine Schicht von der Dicke $\varepsilon$ und der Rotationsintensität $\xi$ liegt. Aus demselben Grunde mufs $v^{\prime}-v_{\alpha}=\mathbf{z} \xi \alpha=\alpha\left(v^{\prime}-v_{1}\right)$ sein, worin der hingestellte Satz liegt. Da wir uns die rotirenden Wassertheilchen selbst als bewegt denken müssen, und die Aenderung der Vertheilung auf der Fläche von ihrer Bewegung abhängt, so müssen wir ihnen als mittlere Geschwindigkeit ihres Fortfliefsens längs der Fläche für die ganze Dicke der Schicht eine solche zuertheilen, welche dem arithmetischen Mittel der an beiden Seiten der Schicht stattindenden Geschwindigkeiten entspricht.

Eine solche Wirbelfläche würde z. B. entstehen, wenn zwei vorher getrennte und bewegte Flüssigkeitsmassen in Berührung mit einander kommen. An der Berührungsfläche würden sich die gegen diese senkrechten Geschwindigkeiten nothwendig ausgleichen müssen. Die sie tangirenden Geschwindigkeiten werden aber im Allgemeinen in den beiden Flüssigkeitsmassen verschieden sein. Die Berührungsfläche würde also die Eigenschaften einer Wirbelfläche haben. 
Dagegen darf man sich im Allgemeinen vereinzelte Wirbelfäden nicht als unendlich dünn denken, weil sonst die Geschwindigkeiten an entgegengesetzten Seiten des Fadens unendlich grofse und entgegengesetzte Werthe erhalten, und die eigene Geschwindigkeit des Fadens deshalb unbestimmt wird. Um nun doch gewisse allgemeine Schlüsse für die Bewegung sehr dünner. Fäden von beliebigem Querschnitt ziehen zu können, wird uns das Princip von der Erhaltung der lebendigen Kraft dienen.

Ehe wir also zu einzelnen Beispielen übergehen, wollen wir noch die Gleichung für die lebendige Kraft $\boldsymbol{K}$ der bewegten Wassermasse bilden:

$$
\text { (6.) } \quad K=\frac{1}{2} h \iiint\left(u^{2}+v^{2}+w^{2}\right) d x d y d z \text {. }
$$

Indern ich in dem Integral nach den Gleichungen (4.) setze

$$
\begin{aligned}
& u^{2}=u\left(\frac{d P}{d x}+\frac{d N}{d y}-\frac{d M}{d z}\right), \\
& v^{2}=v\left(\frac{d P}{d y}+\frac{d L}{d z}-\frac{d N}{d x}\right) \\
& w^{2}=w\left(\frac{d P}{d z}+\frac{d M}{d x}-\frac{d L}{d y}\right)
\end{aligned}
$$

und partiell integrire, dann mit $\cos \alpha, \cos \beta, \cos \gamma$ und $\cos \vartheta$ die Winkel bezeichne, welche die nach innen gerichtete Normale des Elements $d \omega$ der Wassermasse mit den Coordinataxen und der resultirenden Geschwindigkeit $q$ bildet, erhalte ich mit Berücksichtigung der Gleichungen (2.) und (1.) $)_{4}$

$$
\text { (6a.) } \begin{array}{r}
\boldsymbol{K}=-\frac{h}{2} \int d \omega[\boldsymbol{P} q \cos \vartheta+\boldsymbol{L}(v \cos \gamma-w \cos \beta)+\boldsymbol{M}(\boldsymbol{w} \cos \alpha-u \cos \gamma) \\
+N(u \cos \beta-v \cos \alpha)]-h \iiint(\boldsymbol{L} \xi+M \eta+N \zeta) d x d y d z .
\end{array}
$$

Den Werth von $\frac{d K}{d t}$ erhält man aus den Gleichungen (1.), indem man die erste mit $u$, die zweite mit $v$, die dritte mit $v$ multiplicirt und addirt,

$$
\begin{aligned}
h\left(u \frac{d u}{d t}+v \frac{d v}{d t}+w \frac{d w}{d t}\right)= & -\left(u \frac{d p}{d x}+v \frac{d p}{d y}+w \frac{d p}{d z}\right)+h\left(u \frac{d V}{d x}+v \frac{d V}{d y}+v \frac{d V}{d z}\right) \\
& -\frac{h}{2}\left(u \frac{d\left(q^{2}\right)}{d x}+v \frac{d\left(q^{2}\right)}{d y}+w \frac{d\left(q^{2}\right)}{d z}\right) .
\end{aligned}
$$

Wenn man beide Seiten mit $d x d y d z$ multiplicirt, dann über die ganze Ausdehnung der Wassermasse integrirt, und berücksichtigt, dafs wegen (1.)

$$
\iiint\left(u \frac{d \psi}{d x}+v \frac{d \psi}{d y}+w \frac{d \psi}{d z}\right) d x d y d z=-\int \psi q \cos \vartheta d \omega,
$$

wenn $\psi$ im Innern der Wassermasse eine stetige und eindeutige Function 
bezeichnet, so erhält man

$$
\text { (6 b.) } \quad \frac{d K}{d t}=\int d \omega\left(p-h U+\frac{1}{2} h q^{2}\right) q \cos \vartheta .
$$

Wenn die Wassermasse ganz in festen Wänden eingeschlossen ist, mufs $q \cos \vartheta$ an allen Punkten der Oberfläche gleich 0 sein, dann wird also auch $\frac{d K}{d t}=0$, d. h. $K$ constant.

Denkt man sich diese feste Wand in unendlicher Entfernung vom Anfangspunkt der Coordinaten und die vorhandenen Wirbelfäden in endlicher Entfernung, so werden die Potentialfunctionen $\boldsymbol{L}, \boldsymbol{M}, \boldsymbol{N}$, deren Massen $\xi, \eta$, $\zeta$ jede in Summa gleich Null sind, in der unendlichen Entfernung $\Re$ wie $\Re^{-2}$ abnehmen, und die Geschwindigkeiten, ihre Differentialquotienten, wie $\Re^{-3}$, das Flächenelement $d \omega$ aber, wenn es immer dem gleichen Kegelwinkel im Nullpunkte der Coordinaten entsprechen soll, wie $\Re^{2}$ zunehmen. Das erste Integral in dem Ausdrucke für $\boldsymbol{K}$ (Gleichung ( 6 a.)), welches über die Oberfläche der Wassermasse ausgedehnt ist, wird wie $\Re^{-3}$ abnehmen, für ein unendliches $\Re$ also gleich Null werden. Dann reducirt sich der Werth von $\boldsymbol{K}$ auf

$$
\text { (6 c.) } \quad K=-h \iiint(L \xi+M \eta+N \zeta) d x d y d z
$$

und diese Gröfse wird während der Bewegung nicht geändert.

\section{S. 5 .}

\section{Geradlinige parallele Wirbelfäden.}

Wir wollen zuerst den Fall untersuchen, wo nur geradlinige, der Axe der $z$ parallele Wirbelfäden existiren, entweder in einer unendlich ausgedehnten Wassermasse, oder in einer solchen Masse, die durch zwei gegen die Wirbelfäden senkrechte unendliche Ebenen begrenzt ist, was auf dasselbe herauskommt. Alle Bewegungen geschehen dann in Ebenen, die zur Axe der $\approx$ senkrecht sind, und sind in allen diesen Ebenen genau dieselben.

Wir setzen also

$$
w=\frac{d u}{d z}=\frac{d v}{d z}=\frac{d p}{d z}=\frac{d V}{d z}=0 .
$$

Dann reduciren sich die Gleichungen (2.) auf

die Gleichungen (3.) auf

$$
\xi=0, \quad \eta=0, \quad 2 \zeta=\frac{d u}{d y}-\frac{d v}{d x},
$$

$$
\frac{\partial \zeta}{\partial t}=0
$$


Die Wirbelfäden behalten also constante Rotationsgeschwindigkeit, so wie sie auch constanten Querschnitt behalten.

Die Gleichungen (4.) reduciren sich auf

$$
\begin{gathered}
u=\frac{d N}{d y}, \quad v=-\frac{d N}{d x}, \\
\frac{d^{2} N}{d x^{2}}+\frac{d^{2} N}{d y^{2}}=2 \zeta .
\end{gathered}
$$

Ich habe hier nach der am Ende des $\$$. 3 gemachten Bemerkung $\boldsymbol{P}=0$ gesetzt. Die Gleichung der Strömungslinien ist also $N=$ Const.

$\boldsymbol{N}$ ist in diesem Falle die Potentialfunction unendlich langer Linien; diese selbst ist unendlich grofs, aber ihre Differentialquotienten sind endlich. Sind $a$ und $b$ die Coordinaten eines Wirbelfadens, dessen Querschnitt $d a d b$ ist, so ist

$$
-v=\frac{d N}{d x}=\frac{\zeta d a d b}{\pi} \cdot \frac{x-a}{r^{2}}, \quad u=\frac{d N}{d y}=\frac{\zeta d a d b}{\pi} \cdot \frac{y-b}{r^{2}}
$$

Es folgt hieraus, dafs die resultirende Geschwindigkeit $q$ senkrecht gegen $r$, das auf den Wirbelfaden gefällte Loth steht, und dafs

$$
q=\frac{\zeta d a d b}{\pi r} \text {. }
$$

Haben wir in einer in Richtung der $x$ und $y$ unendlich ausgedehnten Wassermasse mehrere Wirbelfäden, deren Coordinaten beziehlich $x_{1}, y_{1}$, $x_{2}, y_{2}$ u. s. w. sind, während das Product aus Rotationsgeschwindigkeit und Querschnitt eines jeden derselben mit $m_{1}, m_{2}$ etc. bezeichnet wird, und bilden wir die Summen

$$
\begin{aligned}
& U=m_{1} u_{1}+m_{2} u_{2}+m_{3} u_{3} \text { etc. } \\
& V=m_{1} v_{1}+m_{2} v_{2}+m_{3} v_{3} \text { etc. }
\end{aligned}
$$

so werden dieselben gleich 0 , weil der Antheil an der Summe $V$, der aus der Wirkung des zweiten Wirbelfadens auf den ersten entsteht, aufgehoben wird durch den vom ersten Wirbelfaden auf den zweiten. Beide sind nämlich

$$
m_{1} \cdot \frac{m_{2}}{\pi} \frac{x_{1}-x_{2}}{r^{2}} \text { und } m_{2} \cdot \frac{m_{1}}{\pi} \frac{x_{2}-x_{1}}{r^{2}}
$$

und so bei allen andern in beiden Summen. Nun ist $\boldsymbol{U}$ die Geschwindigkeit des Schwerpunkts der Massen $m_{1}, m_{2}$ u. s. w. in Richtung der $x$, multiplicirt mit der Summe dieser Massen, ebenso $\boldsymbol{V}$ parallel den $\boldsymbol{y}$ genommen. Beide Geschwindigkeiten sind also gleich Null, wenn nicht die Summe der Massen gleich Null, wo es uberhaupt keinen Sehwerpunkt giebt. Der Schwerpunkt 
der Wirbelfäden bleibt also bei ihrer Bewegung um einander unverändert, und da dieser Satz für jede beliebige Vertheilung der Wirbelfäden gilt, so dürfen wir ihn auch auf einzelne Wirbelfäden von unendlich kleinem Querschnitt anwenden.

Daraus ergeben sich nun nachstehende Folgerungen:

1) Haben wir einen einzelnen geradlinigen Wirbelfaden von unendlich kleinem Querschnitt, in einer nach allen gegen den Wirbelfaden senkrechten Richtungen unendlich ausgedehnten Wassermasse, so hängt die Bewegung der Wassertheilchen in endlicher Entfernung von ihm nur ab von dem Product $\xi d a d b=m$ aus der Rotationsgeschwindigkeit und der Gröfse seines Querschnitts, nicht von der Form seines Querschnitts. Die Theilchen der Wassermasse rotiren um ihn mit der Tangentialgeschwindigkeit $\frac{m}{\pi r}$, wo $r$ die Entfernung vom Schwerpunkte des Wirbelfadens bezeichnet. Die Lage des Schwerpunkts selbst, die Rotationsgeschwindigkeit, die Gröfse des Querschnilts, also auch die Gröfse $m$ bleiben unverändert, wenn auch die Form des unendlich kleinen Querschnitts sich ändern kann.

2) Haben wir zwei geradlinige Wirbelfäden von unendlich kleinem Querschnitt in einer unbegrenzten Wassermasse, so wird jeder den andern in einer Richtung forttreiben, welche senkrecht gegen ihre Verbindungslinie steht. Die Länge der Verbindungslinie wird dadurch nicht geändert. Es werden sich also beide um ihren gemeinschaftlichen Schwerpunkt in gleich bleibendem Abstande drehen. Ist die Rotationsgeschwindigkeit in beiden Wirbelfäden gleich gerichtet, also von gleichem Vorzeichen, so mufs ihr Schwerpunkt zwischen ihnen liegen. Ist sie entgegengesetzt gerichtet, also von ungleichem Vorzeichen, so liegt ihr Schwerpunct in der Verlängerung ihrer Verbindungslinie. Und ist das Product aus der Rotationsgeschwindigkeit und dem Querschnitt bei beiden gleich, aber von entgegengesetztem Zeichen, wobei der Schwerpunkt in unendlicher Entfernung liegen würde, so schreiten sie beide mit gleicher Geschwindigkeit und senkrecht gegen ihre Verbindungslinie in gleicher Richtung fort.

Auf den letzteren Fall kann man auch den zurückführen, wo ein Wirbelfaden von unendlich kleinem Querschnitt sich neben einer ihm parallelen unendlich ausgedehnten Ebene befindet. Die Grenzbedingung für die Bewegung des Wassers an der Ebene, dafs sio der Ebene parallel sein müsse, erfüllt man, indem man jenseits der Ebene noch einen zweiten Wirbelfaden, das 
Spiegelbild des ersten, hinzugefügt denkt. Daraus folgt denn, dafs der in der Wassermasse befindliche Wirbelfaden parallel der Ebene fortschreitet, in der -Richtung, in welcher sich die. Wassertheilchen zwischen ihm und der Ebene bewegen, und mit $\frac{1}{4}$ der Geschwindigkeit, welche die Wassertheilchen im Fufspunkt eines von dem Wirbelfaden auf die Ebene gefällten Lothes haben.

Bei geradlinigen Wirbelfäden führt die Annahme eines unendlich kleinen Querschnitts auf keine unzulässige Folgerung, weil jeder einzelne Faden auf sich selbst keine forttreibende Kraft ausübt, sondern nur durch den Einflufs der anderen vorhandenen Fäden fortgetrieben wird. Anders ist es bei gekrümmten Fäden.

\$. 6 .

Kreisförmige Wirbelfüden.

In einer unendlich ausgedehnten Wassermasse seien nur kreisförmige Wirbelfäden vorhanden, deren Ebenen zur $\approx$ Axe senkrecht sind und deren Mittelpunkte in dieser Axe liegen, so dafs rings um sie herum alles symmetrisch ist. Man ändere die Coordinaten, indem man setzt

$$
\begin{array}{ll}
x=\chi \cos \varepsilon, & a=g \cos e, \\
y=\chi \sin \varepsilon, & b=g \sin e, \\
z=z, & c=c .
\end{array}
$$

Die Rotationsgeschwindigkeit $\sigma$ ist nach der Annahme nur eine Function von $\chi$ und $z$ oder von $g$ und $c$, und die Rotationsaxe steht überall senkrecht auf $\chi$ (oder $g$ ) und der $z$ Axe. Es sind also die rechtwinkligen Componenten der Rotation in dem Punkte, dessen Coordinaten $g, e$ und $c$ sind

$$
\xi=-\sigma \sin e, \quad \eta=\sigma \cos e, \quad \zeta=0 \text {. }
$$

In den Gleichungen ( 5 a.) wird

$$
\begin{aligned}
& r^{2}=(z-c)^{2}+x^{2}+g^{2}-2 x g \cos (\varepsilon-e), \\
& \boldsymbol{L}=\frac{1}{2 \pi} \iiint \frac{\sigma \sin e}{r} g d g d e d c, \\
& \boldsymbol{M}=-\frac{1}{2 \pi} \iiint \frac{\sigma \cos e}{r} g d g d e d c, \\
& \boldsymbol{N}=0 .
\end{aligned}
$$

Indem man mit $\cos \varepsilon$ und $\sin \varepsilon$ multiplicirt und addirt, erhält man aus den Gleichungen für $\boldsymbol{L}$ und $\boldsymbol{M}$

$$
\begin{aligned}
L \sin \varepsilon-M \cos \varepsilon & =-\frac{1}{2 \pi} \iiint \frac{\sigma \cos (\varepsilon-e)}{r} g d g d(\varepsilon-e) d c, \\
L \cos \varepsilon+M \sin \varepsilon & =\frac{1}{2 \pi} \iiint \frac{\sigma \sin (\varepsilon-e)}{r} g d g d(\varepsilon-e) d c
\end{aligned}
$$

Jourual für Mathematik Bd. LV. Heft 1. 
In beiden Integralen kommen die Winkel $e$ und $\varepsilon$ nur noch in der Verbindung $(\varepsilon-e)$ vor, und diese Gröfse kann deshalb als die Variable unter dem Integral betrachtet werden. In dem zweiten Integrale heben sich die Theile, in denen $(\varepsilon-e)=e$ ist, gegen die auf, in denen $(\varepsilon-e)=2 \pi-e$, es wird also gleich Null. Setzen wir

so wird also

(7.) $\psi=\frac{1}{2 \pi} \iiint \frac{\sigma \cos e \cdot g d g d e d c}{\sqrt{(z-c)^{2}+\chi^{2}+y^{2}-2 g \chi \cos e}}$,

oder

$$
\begin{aligned}
& \boldsymbol{M} \cos \varepsilon-\boldsymbol{L} \sin \varepsilon=\psi \\
& \boldsymbol{M} \sin \varepsilon+\boldsymbol{L} \cos \varepsilon=0
\end{aligned}
$$

$$
\text { (7 a.) } \quad \boldsymbol{L}=-\psi \sin \varepsilon, \quad \boldsymbol{M}=\psi \cos \varepsilon \text {. }
$$

Nennen wir $\tau$ die Geschwindigkeit in Richtung des Radius $\chi$, und berücksichtigen, dafs in Richtung der Kreisperipherie wegen der symmetrischen Lage der Wirbelringe zur Axe die Geschwindigkeit gleich Null sein mufs, so haben wir

$$
u=\tau \cos \varepsilon, \quad v=\tau \sin \varepsilon
$$

und nach den Gleichungen (4.)

Daraus folgt

$$
u=-\frac{d M}{d z}, \quad v=\frac{d L}{d z}, \quad w=\frac{d M}{d x}-\frac{d L}{d y} .
$$

oder

$$
\tau=-\frac{d \psi}{d z}, \quad \omega=\frac{d \psi}{d \chi}+\frac{\psi}{\chi},
$$

$$
\text { (7 b.) } \quad \tau_{\chi}=-\frac{d(\psi \chi)}{d z}, \quad w \chi=\frac{d(\psi \chi)}{d \chi} \text {. }
$$

Die Gleichung der Strömungslinien ist also \#

$$
\psi \chi=\text { Const. }
$$

Wenn wir die im Werthe von $\psi$ angezeigte Integration zunächst für einen Wirbelfaden von unendlich kleinem Querschnitt ausführen, dabei setzen $\sigma d g d c=m_{1}$, und den davon herrührenden Theil von $\psi$ mit $\psi_{m_{1}}$ bezeichnen, so ist

$$
\begin{gathered}
\psi_{m_{1}}=\frac{m_{1}}{\pi} \sqrt{\frac{g}{\chi}}\left\{\frac{2}{x}(F-E)-x F\right\}, \\
x^{2}=\frac{4 g x}{(g+x)^{2}+(z-c)^{2}},
\end{gathered}
$$

worin $\boldsymbol{F}$ und $\boldsymbol{E}$ die ganzen elliptischen Integrale erster und zweiter Gattung für den Modul $\varkappa$ bedeuten. 
Setzen wir der Kürze wegen

$$
\boldsymbol{U}=\frac{2}{x}(\boldsymbol{F}-\boldsymbol{E})-x \boldsymbol{F},
$$

wo also $U$ eine Function von $*$ ist, so ist

$$
\tau \chi=\frac{m_{1}}{\pi} \sqrt{g \chi} \frac{d U}{d x} \cdot * \cdot \frac{z-c}{(g+\chi)^{2}+(z-c)^{2}} .
$$

Befindet sich nun in dem durch $\chi$ und $z$ bestimmten Punkte ein zweiter Wirbelfaden $m$, und nennen wir $\tau_{1}$ die Geschwindigkeit in Richtung von $g$, welche er dem Wirbelfaden $m_{1}$ mittheilt, so erhalten wir diese, indem wir in dem Ausdrucke für $\tau$

$\begin{array}{lllllll}\text { statt } & \tau & \chi & g & z & c & m_{1} \\ \text { setzen } & \tau_{1} & g & \chi & c & z & m .\end{array}$

Dabei bleiben $\varkappa$ und $\boldsymbol{U}$ unverändert, und es wird

$$
\text { (8.) } m \tau \chi+m_{1} \tau_{1} g=0 \text {. }
$$

Bestimmen wir nun den Werth der der Axe parallelen Geschwindigkeit $w$, welchen der Wirbelfaden $m_{1}$ hervorbringt, dessen Coordinaten $g$ und $c$ sind, so finden wir

$$
w \chi=\frac{1}{2} \frac{m_{1}}{\pi} \sqrt{\frac{g}{\chi}} U+\frac{m_{1}}{\pi} \sqrt{g \chi} \frac{d U}{d x} \cdot \frac{x}{2 \chi} \cdot \frac{(z-c)^{2}+g^{2}-\chi^{2}}{(g+\chi)^{2}+(z-c)^{2}},
$$

nennt man nun $w_{1}$ die der $\approx$ Axe parallele Geschwindigkeit, welche der Wirbelring $m$, dessen Coordinaten $z$ und $\chi$ sind, am Orte von $m_{1}$ hervorbringt, so braucht man dazu nur wieder die vorher schon angezeigte Vertauschung der betreffenden Coordinaten und Massen vorzunehmen. So findet man, dafs

(8 a.) $2 m w \chi^{2}+2 m_{1} w_{1} g^{2}-m \tau \chi z-m_{1} \tau_{1} g c=\frac{2 m m_{1}}{\pi} \sqrt{g \chi} U$.

Aehnliche Summen wie (8.) und ( 8 a.) lassen sich für eine beliebig grofse Anzahl von Wirbelringen bilden. Ich bezeichne für den $\boldsymbol{n}^{\text {ten }}$ derselben das Product $\sigma d g d c$ mit $m_{n}$, die Componenten der Geschwindigkeit, welche ihm von den übrigen Wirbelringen mitgetheilt werden, mit $\tau_{n}$ und $w_{n}$, wobei aber vorläufig abgesehen wird von den Geschwindigkeiten, die jeder Wirbelring sich selbst mittheilen kann. Ich nenne ferner den Radius des Ringes $\varrho_{n}$ und die Entfernung von einer gegen die Axe senkrechten Fläche $\lambda$, welche beiden letzteren Gröfsen zwar der Richtung nach mit $\chi$ und $z$ übereinstimmen, aber als zu dem bestimmten Wirbelringe gehörig Functionen der Zeit, und nicht unabhängige Variable sind, wie $\chi$ und $z$. Schliefslich sei der Werth 
von $\psi$, so weit dieser von den andern Wirbelringen herrührt $\psi_{n}$. Es ergiebt sich aus (8.) und ( 8 a.), indem man die entsprechenden Gleichungen für jedes einzelne Paar von Wirbelringen aufstellt und alle addirt:

$$
\begin{aligned}
\Sigma\left[m_{n} \varrho_{n} \tau_{n}\right] & =0, \\
\Sigma\left[2 m_{n} w_{n} \varrho_{n}^{2}-m_{n} \tau_{n} \varrho_{n} \lambda_{n}\right] & =\Sigma\left[m_{n} \varrho_{n} \psi_{n}\right] .
\end{aligned}
$$

So lange man in diesen Summen noch eine endliche Zahl getrennter und unendlich dünner Wirbelringe hat, darf man unter $w, \tau$ und $\psi$ nur diejenigen Theile dieser Gröfsen verstehen, welche von der Anwesenheit der anderen Ringe herrühren. Wenn man aber eine unendlich grofse Anzahl solcher Ringe den Raum continuirlich ausfüllend denkt, ist $\psi$ die Potentialfunction einer continuirlichen Masse, $w$ und $\tau$ sind Differentialquotienten dieser Potentialfunction, und es ist bekannt, dafs sowohl in einer solchen Function wie in ihren Differentialquolienten die Theile der Function, welche von der Anwesenheit von Masse in einem unendlich kleinen den betreffenden Punkt, für den die Function bestimmt ist, umgebenden Raum herrühren, unendlich klein sind gegen die von endlichen Massen in endlicher Entfernung herrührenden *):

Verwandeln wir also die Summen in Integrale, so können wir unter $w, \tau$ und $\psi$ die ganzen in dem betreffenden Punkte geltenden Werthe dieser Gröfsen verstehen, und

$$
w=\frac{d \lambda}{d t}, \quad \tau=\frac{d \varrho}{d t}
$$

selzen. Die Gröfse $m$ ersetzen wir zu diesem Zwecke durch das Product $\sigma d \varrho d \lambda$.

$$
\text { (9.) } \iint \sigma \rho \frac{d \varrho}{d t} d \varrho d \lambda=0 \text {, }
$$

(9 a.) $2 \iint \sigma \rho^{2} \frac{d \lambda}{d t} d \varrho d \lambda-\iint \sigma \rho \lambda \frac{d \varrho}{d t} d \rho d \lambda=\iint \sigma \rho \psi d \rho d \lambda$.

Da das Product $\sigma d \varrho d \lambda$ gemäfs $\$ .2$ nach der Zeit constant ist, so kann die Gleichung (9.) nach $t$ integrirt werden, und wir erhalten

$$
\frac{1}{2} \iint \rho^{2} d \rho d \lambda=\text { Const. }
$$

Denkt man den Raum durch eine Ebene getheilt, die durch die $\%$ Axe geht und daher alle vorhandenen Wirbelringe schneidet, betrachten wir dann $\sigma$ als die Dichtigkeit einer Massenschicht, und nennen $\mathfrak{M}$ die ganze in dieser

*) S. Gau/s in Resultate des magnetischen Vereins im Jahre 1839, S. 7. 
Schicht der Ebene anliegende Masse, also

$$
\mathfrak{M}=\iint \sigma d \varrho d \lambda
$$

und $\boldsymbol{R}^{2}$ den mittleren Werth von $\rho^{2}$ für sämmtliche Massenelemente genommen, so ist

$$
\iint \sigma \rho \rho d \rho d \lambda=\mathfrak{M R} \boldsymbol{R}^{2}
$$

und da dieses Integral und der Werth von $\mathfrak{M}$ der Zeit nach constant sind, so folgt, dafs auch $\boldsymbol{R}$ bei der Fortbewegung unverändert bleibt.

Existirt also in der unbegrenzten Flüssigkeitsmasse nur ein kreisförmiger Wirbelfaden von unendlich kleinem Querschnitt, so bleibt dessen Radius unverändert.

Die Gröfse der lebendigen Kraft ist nach Gleichung (6c.) in unserem Falle

$$
\begin{aligned}
\boldsymbol{K} & =-h \iiint(\boldsymbol{L} \xi+\boldsymbol{M} \eta) d a d b d c \\
& =-h \iiint \psi \sigma \cdot \rho d \rho d \lambda d \varepsilon \\
& =-2 \pi h \iint \psi \sigma . \rho d \rho d \lambda .
\end{aligned}
$$

Sie ist ebenfalls der Zeit nach constant.

Indem wir ferner bemerken, dafs, weil $\sigma d \rho d \lambda$ nach der Zeit constant ist

$$
\frac{d}{d t} \iint \sigma \rho^{2} \lambda d \rho d \lambda=2 \iint \sigma \rho \lambda \frac{d \rho}{d t} d \rho d \lambda+\iint \sigma \rho^{2} \frac{d \lambda}{d t} d \lambda d \rho,
$$

so wird die Gleichung ( 9 a.), wenn wir mit $l$ den Werth von $\lambda$ für den Schwerpunkt des Querschnitts des Wirbelfadens bezeichnen, damit (9.) multipliciren und addiren

$$
2 \frac{d}{d t} \iint \sigma \rho^{2} \lambda d \rho d \lambda+5 \iint \sigma \rho(l-\lambda) \frac{d \rho}{d t} d \rho d \lambda=-\frac{K}{2 \pi h} .
$$

Wenn der Querschnitt des Wirbelfadens unendlich klein ist, und $\varepsilon$ eine unendlich kleine Gröfse derselben Orḍnung wie $l-\lambda$ und die übrigen Lineardimensionen des Querschnitts, $\sigma d \rho d \lambda$ aber endlich ist, so ist $\psi$ und auch $K$ von derselben Ordnung unendlich grofser Quantitäten, wie $\log \varepsilon$. Für sehr kleine Werthe des Abstands $v$ vom Wirbelringe wird nämlich

$$
\begin{aligned}
v & =\sqrt{(g-\chi)^{2}+(z-c)^{2}}, \\
x^{2} & =1-\frac{v^{2}}{4 g^{2}}, \\
\psi_{m_{1}} & =\frac{m_{1}}{\pi} \log \left(\frac{\sqrt{1-x^{2}}}{4}\right)=\frac{m_{1}}{\pi} \log \frac{v}{8 g} .
\end{aligned}
$$


In dem Werthe von $\boldsymbol{K}$ wird $\psi$ noch mit $\rho$ oder $\boldsymbol{g}$ multiplicirt. Ist $\boldsymbol{g}$ endlich und $v$ von der gleichen Ordnung mit $\varepsilon$, so ist $\boldsymbol{K}$ von der Ordnung $\log \varepsilon$. Nur wenn $g$ unendlich grofs von der Ordnung $\frac{1}{\varepsilon}$ ist, wird $K$ unendlich grofs, wie $\frac{1}{\varepsilon} \log \varepsilon$. Dann geht der Kreis in eine gerade Linie über. Dagegen wird $\frac{d \varrho}{d t}$, welches gleich $\frac{d \psi}{d z}$ ist, von der Ordnung $\frac{1}{\varepsilon}$, das zweite Integral also endlich und bei endlichem $\rho$ verschwindend klein gegen $\boldsymbol{K}$. In diesem Falle können wir im ersten Integrale das constante $l$ statt $\lambda$ setzen, und erhalten

oder

$$
2 \frac{d\left(\mathfrak{R} R^{2} l\right)}{d t}=-\frac{K}{2 \pi h}
$$

$$
2 \mathfrak{M R} R^{2} l=C-\frac{K}{2 \pi h} t \text {. }
$$

Da $\mathfrak{M}$ und $\boldsymbol{R}$ constant sind, kann sich nur $\boldsymbol{l}$ proportional der Zeit ändern. Wenn $\mathfrak{M}$ positiv ist, ist die Bewegung der Wassertheilchen auf der äufsern Seite des Ringes nach der Seite der positiven $z$, auf der innern nach der der negativen $z$ gerichtet; $\boldsymbol{K}, \boldsymbol{h}$ und $\boldsymbol{R}$ sind ihrer Natur nach immer positiv.

Daraus folgt also, da/s bei einem kreisförmigen Wirbelfaden von sehr kleinem Querschnitt in einer unendlich ausgedehnten Wassermasse der Schwerpunkt des Querschnitts eine der Axe des Wirbelringes parallele Bewegung hat von annühernd constanter und sehr gro/ser Geschwindigkeit, die nach derselben Seite hin gerichtet ist, nach welcher das Wasser durch den Ring strömt. Unendlich dünne Wirbelfäden von endlichem Radius würden unendlich grofse Fortpflanzungsgeschwindigkeit erhalten. Ist aber der Radius des Wirbelrings unendlich grofs von der Ordnung $\frac{1}{\varepsilon}$, so wird $\boldsymbol{R}^{2}$ unendlich grofs gegen $\boldsymbol{K}$, und $l$ wird constant. Der Wirbelfaden, welcher sich nun in eine gerade Linie verwandelt hat, wird stationär, wie wir für geradlinige Wirbelfäden schon früher gefunden haben.

Es läfst sich nun auch im Allgemeinen übersehen, wie sich zwei ringförmige Wirbelfäden, deren Axe dieselbe ist, gegen einander verhalten werden, da jeder abgesehen von seiner eigenen Fortbewegung auch der Bewegung der Wassertheilchen folgt, die der andere hervorbringt. Haben sie gleiche Rotationsrichtung, so schreiten sie beide in gleichem Sinne fort, und es wird der vorangehende sich erweitern, dann langsamer fortschreiten, der nachfolgende sich verengern und schneller. fortschreiten, schliefslich bei nicht $\mathrm{zu}$ differenten Fortpflanzungsgeschwindigkeiten den andern einholen, durch ihn 
hindurchgehen. Dann wird sich dasselbe Spiel mit dem andern wiederholen, so dafs die Ringe abwechselnd einer durch den andern hindurchgehen.

Haben die Wirbelfäden gleiche Radien, gleiche und entgegengesetzte Rotationsgeschwindigkeiten, so .werden sie sich einander nähern, und sich gegenseitig erweitern, so dafs schliefslich, wenn sie sich sehr nah gekommen sind, ihre Bewegung gegen einander immer schwächer wird, die Erweiterung dagegen mit wachsender Geschwindigkeit geschieht. Sind die beiden Wirbelfäden ganz symmetrisch, so ist in der Mitte zwischen beiden die der Axe parallele Geschwindigkeit der Wassertheilchen gleich Null. Man kann sich hier also eine feste Wand angebracht denken, ohne die Bewegung zu stören, und erhält so den Fall eines Wirbelringes, der gegen eine feste Wand anläuft.

Ich bemerke noch, dafs man diese Bewegungen der kreisförmigen Wirbelringe in der Natur leicht studiren kann, indem man eine halb eingetauchte Kreisscheibe, oder die ungefähr halbkreisförmig begrenzte Spitze eines Löffels schnell eine kurze Strecke längs der Oberfläche der Flüssigkeit hinführt, und dann schnell herauszieht. Es bleiben dann halbe Wirbelringe in der Flüssigkeit zurück, deren Axe in der freien Oberfläche liegt. Die freie Oberfläche bildet also eine durch die Axe gelegte Begrenzungsebene der Wassermasse, wodurch an den Bewegungen nichts wesentliches geändert wird. Die Wirbelringe schreiten fort, erweitern sich, wenn sie gegen eine Wand laufen, und werden durch andere Wirbelringe erweitert oder verengert, ganz wie wir es aus der Theorie abgeleitet haben. 